\title{
Weak Nontrivial Solutions to Discrete Nonlinear Two-Point Boundary-Value Problems of Kirchhoff Type
}

\author{
Rodrigue $\mathrm{SANOU}^{1}$, Idrissa IBRANGO${ }^{2}$ and Blaise $\mathrm{KONE}^{3 *}$ \\ ${ }^{1}$ Laboratoire de Mathématiques et Informatique (LAMI), UFR, Sciences Exactes et Appliquées, \\ Université Joseph-Ki-ZERBO, 03 BP 7021 Ouaga 03, Ouagadougou, Burkina-Faso. \\ ${ }^{2}$ Laboratoire de Mathématiques et Informatique (LAMI), UFR, Sciences et Technique, \\ Université Nazi BONI, 01 BP 1091 Bobo 01, Bobo Dioulasso, Burkina-Faso. \\ ${ }^{3}$ Laboratoire de Mathématiques et Informatique (LAMI), Institut Burkinabé des Arts et Métiers, \\ Université Joseph-Ki-ZERBO, 03 BP 7021 Ouaga 03, Ouagadougou, Burkina-Faso. \\ Email: ${ }^{1}$ drigoaime@gmail.com, ${ }^{2}$ ibrango2006@yahoo.fr, ${ }^{3}$ leizon71@yahoo.fr
}

\begin{abstract}
We prove the existence of at least one weak nontrivial solutions for a discrete nonlinear two-point boundary-value problems of Kirchhoff type. The main existence results are obtained by using the technique of geometric mountain pass and the Ekelands variational principle.
\end{abstract}

2010 Mathematics Subject Classification: 47A75; 35B38; 35P30; 34L05; 34L30.

Keywords: discrete boundary value problem, critical point, weak solution, mountain pass geometry lemma, Palais-Smale condition.

\section{Introduction}

In this paper, we investigate the existence of at least one weak nontrivial solutions for the discrete nonlinear problems of Kirchhoff type.

$$
\left\{\begin{array}{l}
-M(A(k-1, \Delta u(k-1))) \Delta(a(k-1, \Delta u(k-1)))=\lambda f(k, u(k)), k \in \mathbb{N}[1, T] \\
u(0)=\Delta u(T)=0,
\end{array}\right.
$$

where $\lambda$ is a numerical parameter. $\Delta u(k)=u(k+1)-u(k)$ is the forward difference operator, $\mathbb{N}[1, T]=$ $\{1, \ldots, T\}, a, A, M$ and $f$ are functions to be defined later (see $[5,13]$ ).

Discrete boundary value problems and nonlinear difference equations emerge from real world problems and are claimed to be employed as handy means for the description of the processes which are endowed with discrete intervals. They intervene in many fields such as economy, biology, physics, mechanics, computer science and finance $[1,2]$.

Problem (1.1) has its origin in the theory of nonlinear vibration. For instance, the following equation describes the free vibration of a stretched string (see [3])

$$
\rho \frac{\partial^{2} u}{\partial t^{2}}=\left(T_{0}+\frac{E a}{2 L} \int_{0}^{L}\left|\frac{\partial u}{\partial x}\right|^{2} d x\right) \frac{\partial^{2} u}{\partial x^{2}}
$$

where $\rho>0$ is the mass per unit length, $T_{0}$ is the base tension, $E$ is the Young modulus, $a$ is the area of cross section and $L$ is the initial length of the string. In [4] Blaise Kone and Stanislas Ouaro are studying the following problem

$$
\begin{gathered}
-\Delta(a(k-1, \Delta u(k-1)))=f(k), k \in \mathbb{N}[1, T] \\
u(0)=\Delta u(T)=0,
\end{gathered}
$$

where $T \geq 2$ is positive integer. In [5] the authors proceed to the generalization of the probem (1.3). In our paper we base ourselves on the model of the [5] taking $f(k, u(k))$ instead of $f(k)$. The major 
difference between the [5] and our problem (1.1) lies in the fact that by four different methods we prove that the problem (1.1) admits at least a weak nontivial solution.

Our paper is organized in the following way. In section2 we define the general results that we will use throughout our work. In section3 we show that problem (1.1) admits at least a weak nontrivial solution. In section4 we proceed to an extension of the previous results.

\section{Mathematical Background}

By a solution to problem (1.1) we mean such a function $u: \mathbb{N}[0, T+1] \longrightarrow \mathbb{R}$ which satisfies the given equation on $\mathbb{N}[1, T]$ and the boundary conditions. In the $T$-dimensional Hilbert space

$$
X=\{u: \mathbb{N}[0, T+1] \longrightarrow \mathbb{R}: u(0)=\Delta u(T)=0\}
$$

with the inner product

$$
\langle u, v\rangle=\sum_{k=1}^{T} u(k) v(k), \quad \forall u, v \in X
$$

we consider the norm

$$
\|u\|=\left(\sum_{k=1}^{T}|u(k)|^{2}\right)^{\frac{1}{2}}
$$

Let the function

$$
p: \mathbb{N}[0, T] \longrightarrow[2,+\infty)
$$

and denoted by

$$
p^{-}=\min _{k \in \mathbb{N}[0, T]} p(k), \quad p^{+}=\max _{k \in \mathbb{N}[0, T]} p(k) .
$$

For the data $a$ and $f$, we assume the following.

$\left(H_{1}\right) . \quad\left\{\begin{array}{c}a(k, .): \mathbb{R} \rightarrow \mathbb{R}, \quad k \in \mathbb{N}[0, T] \text { and there exists } A(., .): \mathbb{N}[0, T] \times \mathbb{R} \rightarrow \mathbb{R} \\ \text { which satisfies } a(k, \xi)=\frac{\partial}{\partial \xi} A(k, \xi) \text { and } A(k, 0)=0, \quad \text { for all } k \in \mathbb{N}[0, T] .\end{array}\right.$

$\left(H_{2}\right)$. For all $k \in \mathbb{N}[0, T]$ and $\xi \neq \eta$

$$
(a(k, \xi)-a(k, \eta)) \cdot(\xi-\eta)>0 .
$$

$\left(H_{3}\right)$. For any $k \in \mathbb{N}[0, T], \xi \in \mathbb{R}$, we have

$$
p(k) A(k, \xi) \geq a(k, \xi) \xi \geq|\xi|^{p(k)} .
$$

$\left(H_{4}\right)$. For any $k \in \mathbb{N}[0, T], \xi \in \mathbb{R}$ it exist $C_{1}>0$ such that

$$
|a(k, \xi)| \leq C_{1}\left(1+|\xi|^{p(k)-1}\right) .
$$

$\left(H_{5}\right)$. We also assume that the function $M:(0,+\infty) \longrightarrow(0,+\infty)$ is continuous and non-decreasing and there exist positive numbers $D_{1}, D_{2}$ with $D_{1} \leq D_{2}$ and $\quad \alpha>1$ such that

$$
D_{1} t^{\alpha-1} \leq M(t) \leq D_{2} t^{\alpha-1} \quad \text { for } \quad t>t^{*}>0 .
$$

$\left(H_{6}\right)$. For each $k \in \mathbb{N}[1, T]$, the function $f(k,):. \mathbb{R} \longrightarrow \mathbb{R}$ is jointly continuous and there exists the functions $A_{1}, A_{2}: \mathbb{N}[1, T] \longrightarrow \mathbb{R}^{-} \backslash\{0\} ; B_{1}, B_{2}: \mathbb{N}[1, T] \longrightarrow(0,+\infty)$ and a function $r: \mathbb{N}[1, T] \longrightarrow$ $[2,+\infty)$ such that

$$
A_{1}(k)+B_{1}(k)|\xi|^{r(k)-1} \leq f(k, \xi) \leq B_{2}(k)|\xi|^{r(k)-1}+A_{2}(k)
$$


where

$$
\begin{aligned}
& -\infty<\underline{A_{1}}=\inf _{k \in \mathbb{N}[1, T]} A_{1}(k), \quad \overline{A_{1}}=\sup _{k \in \mathbb{N}[1, T]} A_{1}(k)<0 ; \\
& -\infty<\underline{A_{2}}=\inf _{k \in \mathbb{N}[1, T]} A_{2}(k), \quad \overline{A_{2}}=\sup _{k \in \mathbb{N}[1, T]} A_{2}(k)<0 ; \\
& 0<\underline{B_{1}}=\inf _{k \in \mathbb{N}[1, T]} B_{1}(k), \quad \overline{B_{1}}=\sup _{k \in \mathbb{N}[1, T]} B_{1}(k)<+\infty ; \\
& 0<\underline{B_{2}}=\inf _{k \in \mathbb{N}[1, T]} B_{2}(k), \quad \overline{B_{2}}=\sup _{k \in \mathbb{N}[1, T]} B_{2}(k)<+\infty ; \\
& r^{-}=\min _{k \in \mathbb{N}[1, T]} r(k), \quad r^{+}=\max _{k \in \mathbb{N}[1, T]} r(k) \text { and there exists } \\
& \alpha_{1}, \alpha_{2}>1 \text { such that } \underline{B_{1}}>\max \left(\alpha_{1}, \alpha_{2}\right)\left|\underline{A_{1}}\right| r^{+} .
\end{aligned}
$$

We denote

$$
F(k, \xi)=\int_{0}^{\xi} f(k, s) d s \text { for }(k, \xi) \in \mathbb{N}[0, T] \times \mathbb{R} .
$$

Example 2.1. There are many functions satisfying both $\left(H_{1}\right)-\left(H_{5}\right)$. Let us mention the following.

$$
\begin{aligned}
& -A(k, \xi)=\frac{1}{p(k)}\left(\left(1+|\xi|^{2}\right)^{p(k) / 2}-1\right), \text { where } a(k, \xi)=\left(1+|\xi|^{2}\right)^{(p(k)-2) / 2} \xi, \quad \forall k \in \mathbb{N}[0, T], \xi \in \mathbb{R}, \\
& -f(k, \xi)=1+|\xi|^{r(k)-1}, \quad \forall k \in \mathbb{N}[1, T] \text { and } \xi \in \mathbb{R}^{+}, \\
& -M(t)=1 .
\end{aligned}
$$

Moreover, we may consider $X$ with the following norm

$$
|u|_{m}=\left(\sum_{k=1}^{T}|u(k)|^{m}\right)^{\frac{1}{m}}, \quad \forall u \in X \quad \text { and } \quad m \geq 2 .
$$

We have the following inequalities (see [6])

$$
T^{(2-m) /(2 m)}|u|_{2} \leq|u|_{m} \leq T^{1 / m}|u|_{2}, \quad \forall u \in X \quad \text { and } \quad m \geq 2 .
$$

We need the following auxiliary results throughout our paper (see [7]).

\section{Lemma 2.1.}

1. There exist two positive constant $C_{2}, C_{3}$ such that

$$
\sum_{k=1}^{T+1}|\Delta u(k-1)|^{p(k-1)} \geq C_{2}\left(\sum_{k=1}^{T+1}|\Delta u(k-1)|^{2}\right)^{\frac{p^{-}}{2}}-C_{3}
$$

for all $u \in X$ with $\|u\|>1$.

2. For every $u \in X$ with $\|u\| \leq 1$ we have

$$
\sum_{k=1}^{T+1}|\Delta u(k-1)|^{p(k-1)} \geq T^{-\frac{p^{+}-2}{2}}\left(\sum_{k=1}^{T+1}|\Delta u(k-1)|^{2}\right)^{\frac{p^{+}}{2}} .
$$

3. For any $m \geq 2$ there exists a positive constant $c_{m}$ such that

$$
\sum_{k=1}^{T}|u(k)|^{m} \leq c_{m} \sum_{k=1}^{T+1}|\Delta u(k-1)|^{m}, \quad \forall u \in X .
$$


4. For every $u \in X$ we have

$$
\sum_{k=1}^{T+1}|\Delta u(k-1)|^{p(k-1)} \leq(T+1)\left(2^{p^{+}}\|u\|^{p^{+}}+1\right)
$$

(see [8]).

Theorem 2.1. [9] Let $H$ be a reflexive Banach space. If a functioal $J \in C^{1}(H, \mathbb{R})$ is weakly lower semicontinuous and coercive, i.e. $\lim _{\|x\| \rightarrow \infty} J(x)=+\infty$, then there exists $x_{0}$ such that

$$
\inf _{x \in H} J(x)=J\left(x_{0}\right)
$$

and $x_{0}$ is also a critical point of $J$, i.e. $J^{\prime}\left(x_{0}\right)=0$. Moreover, if $J$ is strictly convex, then a critical point is unique.

Theorem 2.2. [10](Ekeland's principle) Let $E$ be a complete metric space and $\Phi: E \longrightarrow \mathbb{R}$ a lower semicontinuous function that is bounded below. Let $\varepsilon>0$ and $\bar{u} \in E$ be given such that

$$
\Phi(\bar{u}) \leq \inf _{E} \Phi+\frac{\varepsilon}{2}
$$

Then given $\lambda>0$ there exists $u_{\lambda} \in E$ such that

(i) $\Phi\left(u_{\lambda}\right) \leq \Phi(\bar{u})$

(ii) $d\left(u_{\lambda}, \bar{u}\right)<\lambda$,

(iii) $\Phi\left(u_{\lambda}\right)<\Phi(u)+\frac{\varepsilon}{\lambda} d\left(u, u_{\lambda}\right)$ for all $u \neq u_{\lambda}$.

Definition 2.1. Let $H$ be a real Banach space. We say that a functional $J: H \longrightarrow \mathbb{R}$ satisfies the PalaisSmale condition if every sequence $\left(u_{n}\right)$ such that $\left\{J\left(u_{n}\right)\right\}$ is bounded and $J^{\prime}\left(u_{n}\right) \rightarrow 0$ has a convergent subsequence.

Lemma 2.2. [11] Let $H$ be a Banach space and $J \in C^{1}(H, \mathbb{R})$ satisfies the Palais-Smale condition. Assume that there exist $x_{0}, x_{1} \in H$ and a bounded open neighborhood $\Omega$ of $x_{0}$ such that $x_{1} \notin \bar{\Omega}$ and

$$
\max \left\{J\left(x_{0}\right), J\left(x_{1}\right)\right\}<\inf _{x \in \partial \Omega} J(x) .
$$

Let

$$
\Gamma=\left\{h \in C([0,1], H): h(0)=x_{0}, \quad h(1)=x_{1}\right\}
$$

and

$$
c=\inf _{h \in \Gamma} \max _{s \in[0,1]} J(h(s)) .
$$

Then $c$ is a critical value of $J$; that is, there exists $x^{*} \in H$ such that $J^{\prime}\left(x^{*}\right)=0$ and $J\left(x^{*}\right)=c$, where $c>\max \left\{J\left(x_{0}\right), J\left(x_{1}\right)\right\}$.

Theorem 2.3. [12] Let $E$ be a finite-dimensional Euclidean space, $\eta, \mu_{1}, \mu_{2}: E \longrightarrow \mathbb{R}$ be differentiable function, and $S=\left\{x \in E: \mu_{1} \leq 0, \mu_{2} \leq 0\right\}$. Moreover, let $\bar{x} \in S$ be such that $\eta(\bar{x})=\inf _{S} \eta(x)$. Then there exist numbers $\sigma_{0}, \sigma_{1}, \sigma_{2} \geq 0$ such that $\left(\sigma_{0}\right)^{2}+\left(\sigma_{1}\right)^{2}+\left(\sigma_{2}\right)^{2}>0$ and

$$
\sigma_{0} \eta^{\prime}(\bar{x})+\sigma_{1} \mu_{1}^{\prime}(\bar{x})+\sigma_{2} \mu_{2}^{\prime}(\bar{x})=0 \quad \text { and } \quad \sigma_{1} \mu_{1}(\bar{x})=0, \quad \sigma_{2} \mu_{2}(\bar{x})=0 .
$$

\section{Existence of a Solution}

In this part we will show that the problem (1.1) admits at least one nontrivial solution. For that we will use several methods such as that of minimization, the method of montain pass then that of Ekelands variational principle. First of all we will check that the functional $J_{\lambda}$ satisfies the condition of PalaisSmale. 
We define the energy functional corresponding to $(1.1) ; J_{\lambda}: X \longrightarrow \mathbb{R}$ by

$$
J_{\lambda}(u)=\widehat{M}\left(\sum_{k=1}^{T+1} A(k-1, \Delta u(k-1))\right)-\lambda \sum_{k=1}^{T} F(k, u(k))
$$

where $\widehat{M(t)}=\int_{0}^{t} M(s) d s$.

The functional $J_{\lambda}$ is differentiable in sense of Gâteaux and its Gâteaux derivative reads

$$
M\left(\sum_{k=1}^{T+1} A(k-1, \Delta u(k-1))\right) \sum_{k=1}^{T+1} a(k-1, \Delta u(k-1)) \Delta v(k-1)=\lambda \sum_{k=1}^{T} f(k, u(k)) v(k),
$$

for all $u, v \in X$.

A critical point to $J_{\lambda}$, i.e. a point $u \in X$ such that

$$
\left\langle J_{\lambda}^{\prime}(u), v\right\rangle=0 \quad \text { for all } \quad v \in X
$$

is a weak solution to (1.1).The previous results have been prouven in $[5,13,14]$.

Lemma 3.1. Assume that (2.5), (2.6), (2.7),(2.10) holds and $r^{-}>p^{+} \alpha$. Then for any $\lambda>0$ the functional $J_{\lambda}$ satisfies the Palais-Smale condition.

\section{Proof}

Assume that $\left\{u_{n}\right\}$ is such that $\left\{J_{\lambda}\left(u_{n}\right)\right\}$ is bounded and $J_{\lambda}^{\prime}\left(u_{n}\right) \rightarrow 0$.

We have $X$ finitely dimensional, it is enough to show that $\left\{u_{n}\right\}$ is bounded. Assume that $\left\{u_{n}\right\}$ is unbounded, we have for $n$ large enough, $\left\|u_{n}\right\|$ sufficiently large. We consider $n$ large enough by (2.5), $(2.6),(2.7)$ and $(2.10)$

$$
\begin{aligned}
& J_{\lambda}\left(u_{n}\right) \leq \frac{D_{2}}{\alpha}\left(\sum_{k=1}^{T+1} A\left(k-1, \Delta u_{n}(k-1)\right)^{\alpha}-\lambda\left(-\left|\underline{A_{1}}\right| \sum_{k=1}^{T}\left|u_{n}(k)\right|+\frac{B_{1}}{r^{+}} \sum_{k=1}^{T}\left|u_{n}(k)\right|^{r(k)}\right)\right. \\
& \leq \frac{D_{2}}{\alpha}\left[\sum_{k=1}^{T+1} \int_{0}^{\Delta u_{n}(k-1)}|a(k-1, s)| d s\right]^{\alpha}-\lambda\left(-\left|\underline{A_{1}}\right| \sqrt{T} \sum_{k=1}^{T}\left|u_{n}(k)\right|^{2}+\frac{B_{1}}{\overline{r^{+}}} \sum_{k=1}^{T}\left|u_{n}(k)\right|^{r^{-}}\right) \\
& \leq \frac{D_{2} C_{1}^{\alpha}}{\alpha}\left[\sum_{k=1}^{T+1}\left(\left|\Delta u_{n}(k-1)\right|+\frac{\left|\Delta u_{n}(k-1)\right|^{p(k-1)}}{p^{-}}\right)\right]^{\alpha}- \\
& \lambda\left(-\underline{A_{1}}|\sqrt{T}| \mid u_{n}\left\|^{2}+\frac{B_{1} T^{\frac{2-r^{-}}{2}}}{r^{+}}\right\| u_{n} \|^{r^{-}}\right) \\
& \leq \frac{D_{2} C_{1}^{\alpha}}{\alpha}\left[\left|u_{n}(T+1)\right|+2 \sqrt{T}\left\|u_{n}\right\|+\frac{(T+1)\left(2^{p^{+}}\left\|u_{n}\right\|^{p^{+}}+1\right)}{p^{-}}\right]^{\alpha}- \\
& \lambda\left(-\underline{A_{1}}|\sqrt{T}| \mid u_{n}\left\|^{2}+\frac{B_{1} T^{\frac{2-r^{-}}{2}}}{r^{+}}\right\| u_{n} \|^{r^{-}}\right) .
\end{aligned}
$$

Since $r^{-}>p^{+} \alpha$ and $\left\|u_{n}\right\| \rightarrow+\infty$; we have $J_{\lambda}\left(u_{n}\right) \rightarrow-\infty$. This is absurd. So the sequence $\left\{u_{n}\right\}$ is bounded.

\subsection{Case $p^{-}>\frac{r^{+}}{\alpha}$}

In this part by the method of minimization, we will show that the problem (1.1) admits at least a weak nontrivial solution. 
Proposition 3.1. Assume that (2.4),(2.6), (2.7),(2.10), (2.11), (2.13) holds and $p^{-}>\frac{r^{+}}{\alpha}, J_{\lambda} i s$ coercive for all $\lambda>0$.

Proof according to (2.4), (2.6) and (2.7), we have

$$
\begin{aligned}
J_{\lambda}(u) & =\widehat{M}\left(\sum_{k=1}^{T+1} A(k-1, \Delta u(k-1))\right)-\lambda \sum_{k=1}^{T} F(k, u(k)) \\
& \geq \frac{D_{1}}{\alpha}\left[\sum_{k=1}^{T+1} A(k-1, \Delta u(k-1))\right]^{\alpha}-\lambda\left(\frac{\overline{B_{2}}}{r^{-}} \sum_{k=1}^{T}|u(k)|^{r(k)}+\left|\underline{A_{2}}\right| \sum_{k=1}^{T}|u(k)|\right) \\
& \geq \frac{D_{1}}{\alpha\left(p^{+}\right)^{\alpha}}\left[\sum_{k=1}^{T+1}|\Delta u(k-1)|^{p(k-1)}\right]^{\alpha}-\lambda\left(\frac{\overline{B_{2}}}{r^{-}} \sum_{k=1}^{T}|u(k)|^{r(k)}+\left|\underline{A_{2}}\right| \sum_{k=1}^{T}|u(k)|\right) .
\end{aligned}
$$

We will prouve the coercivity of $J_{\lambda}$ for $\|u\|>1$. We deduce from the above inequality $(2.10),(2.11)$ and (2.13) that

$$
\begin{aligned}
J_{\lambda}(u) & \geq \frac{D_{1}}{\alpha\left(p^{+}\right)^{\alpha}}\left[C_{2}\left(\sum_{k=1}^{T+1}|\Delta u(k-1)|^{2}\right)^{\frac{p^{-}}{2}}-C_{3}\right]^{\alpha}- \\
& \lambda\left(\frac{\overline{B_{2}}}{r^{-}} \sum_{k=1}^{T}|u(k)|^{r^{+}}+\frac{\overline{B_{2}}}{r^{-}} \sum_{k=1}^{T}|u(k)|^{r^{-}}+\left|\underline{A_{2}}\right| \sqrt{T} \sum_{k=1}^{T}|u(k)|^{2}\right) \\
& \geq \frac{D_{1}}{\alpha\left(p^{+}\right)^{\alpha}}\left[\frac{C_{2}}{\left(c_{2}\right)^{\frac{p^{-}}{2}}}|| u||^{p^{-}}-C_{3}\right]^{\alpha}-\lambda\left(\frac{\overline{B_{2}}}{r^{-}} T|| u\left\|^{r^{+}}+\frac{\overline{B_{2}}}{r^{-}} T\right\| u\left\|^{r^{-}}+\left|\underline{A_{2}}\right| \sqrt{T}|| u\right\|^{2}\right) .
\end{aligned}
$$

Hence $p^{-}>\frac{r^{+}}{\alpha}, J_{\lambda}$ is coercive.

Theorem 3.1. Assume that (2.5) and (2.7) holds, for all $p^{-}>\frac{r^{+}}{\alpha}$ and $\lambda>\lambda_{0}$, the problem (1.1) has at least one weak nontrivial solution.

Proof. By $[5] J_{\lambda} \in C^{1}(X, \mathbb{R})$ and weakly lower semicontinuous. Moreover by Proposition 3.1 we prove the Theorem 2.1. Let $u_{\varepsilon} \in X$ a global minimizer of $J_{\lambda}$ which is a weak solution of problem (1.1).

We show $u_{\varepsilon}$ is not trivial for all $\alpha p^{-}>r^{+}$and $\lambda>\lambda_{0}$.

For $t_{0}>1$ be a fixed real and $k_{0} \in \mathbb{N}[0, T+1]$, we define $u_{0}: \mathbb{N}[0, T+1] \longrightarrow \mathbb{R}$ such that $u_{0}\left(k_{0}\right)=t_{0}, u_{0}(k)=0 \quad$ for any $k \in \mathbb{N}[0, T] \backslash\left\{k_{0}\right\} \quad$ and $\quad u_{0}(0)=\Delta u_{0}(T)=0$, we have $u_{0} \in X$.

By (2.5), (2.6) and (2.7)

$$
\begin{aligned}
J_{\lambda}\left(u_{0}\right) & \leq \frac{D_{2} C_{1}^{\alpha}}{\alpha}\left(2 t_{0}+\frac{t_{0}^{p\left(k_{0}-1\right)}+t_{0}^{p\left(k_{0}\right)}}{p^{-}}\right)^{\alpha}-\lambda\left(-\underline{A_{1}} \mid t_{0}+\frac{B_{1}}{\overline{r^{+}}} t_{0}^{r^{-}}\right) \\
& \leq \frac{D_{2}\left(4 C_{1}\right)^{\alpha} t_{0}^{\alpha p^{+}}}{\alpha}-\lambda t_{0}\left(-\left|\underline{A_{1}}\right|+\frac{B_{1}}{r^{+}}\right) \\
& \leq \frac{D_{2}\left(4 C_{1}\right)^{\alpha} t_{0}^{\alpha p^{+}}}{\alpha}-\lambda t_{0}\left(-\max \left(\alpha_{1}, \alpha_{2}\right)\left|\underline{A_{1}}\right|+\frac{B_{1}}{\overline{r^{+}}}\right)
\end{aligned}
$$

where

$$
\lambda_{0}=\frac{D_{2}\left(4 C_{1}\right)^{\alpha} t_{0}^{\alpha p^{+}-1}}{\alpha\left(-\max \left(\alpha_{1}, \alpha_{2}\right)\left|\underline{A_{1}}\right|+\frac{B_{1}}{r^{+}}\right)} .
$$

We have $J_{\lambda}\left(u_{0}\right)<0$ for any $\left.\left.\lambda \in\right] \lambda_{0},+\infty\right)$. It follows that $J_{\lambda}\left(u_{\varepsilon}\right)<0$ for any $\lambda>\lambda_{0}, u_{\varepsilon}$ is a weak nontrivial solution of problem (1.1) for $\lambda$ large enough. 


\section{2 $\quad$ Case $r^{-}>p^{+} \alpha$}

We have previously shown that if $r^{-}>p^{+} \alpha \quad J_{\lambda}$ satisfies the Palais-Smale condition. We can thus use the mountain pass geometry Lemma.

Theorem 3.2. Let $r^{-}>p^{+} \alpha$ and the condition (2.4), (2.5), (2.6), (2.7), (2.10), (2.12), (2.13) holds. Then for $\lambda \in\left(0, \lambda_{1}\right)$ the problem (1.1) has at least one weak nontrivial solution.

Proof. Let

$$
\Omega=\{u \in X:\|u\| \leq \theta\}
$$

with $\theta \in(0,1)$.

For $u \in \Omega$, by (2.4), (2.6), (2.7), (2.10), (2.12) and (2.13)

$$
\begin{aligned}
J_{\lambda}(u) & \geq \frac{D_{1}}{\alpha\left(p^{+}\right)^{\alpha}}\left[\sum_{k=1}^{T+1}|\Delta u(k-1)|^{p(k-1)}\right]^{\alpha}-\lambda\left(\frac{\overline{B_{2}}}{r^{-}} \sum_{k=1}^{T}|u(k)|^{r(k)}+\left|\underline{A_{2}}\right| \sum_{k=1}^{T}|u(k)|\right) \\
& \geq \frac{D_{1} T^{-\frac{\left(p^{+}-2\right) \alpha}{2}}}{\alpha\left(p^{+}\right)^{\alpha}}\left(\sum_{k=1}^{T+1}|\Delta u(k-1)|^{2}\right)^{\frac{p^{+} \alpha}{2}}-\lambda\left(\frac{\overline{B_{2}}}{r^{-}} \sum_{k=1}^{T}|u(k)|^{r^{-}}+\left|\underline{A_{2}}\right| \sqrt{T}\left(\sum_{k=1}^{T}|u(k)|^{2}\right)^{\frac{1}{2}}\right) \\
& \geq \frac{D_{1} T^{-\frac{\left(p^{+}-2\right) \alpha}{2}}}{\alpha\left(c_{2}\right)^{\frac{\alpha p^{+}}{2}}\left(p^{+}\right)^{\alpha}}|| u \|^{p^{+} \alpha}-\lambda\left(\left.\frac{\overline{B_{2}} T}{r^{-}}|| u\right|^{r^{-}}+\left|\underline{A_{2}}\right| \sqrt{T}|| u \|\right) .
\end{aligned}
$$

For $u \in \partial \Omega$, we obtain

$$
J_{\lambda}(u) \geq \frac{D_{1} T^{-\frac{\left(p^{+}-2\right) \alpha}{2}}}{\alpha\left(c_{2}\right)^{\frac{\alpha p^{+}}{2}}\left(p^{+}\right)^{\alpha}} \theta^{\alpha p^{+}}-\lambda \theta\left(\frac{\overline{B_{2}} T}{r^{-}}+\left|\underline{A_{2}}\right| \sqrt{T}\right) .
$$

So for everything $\lambda \in\left(0, \lambda_{1}\right)$

$$
J_{\lambda}(u)>0 \text { for all } u \in \partial \Omega
$$

with

$$
\lambda_{1}=\frac{\frac{\theta^{\left(\alpha p^{+}-1\right)} D_{1} T^{-}}{\frac{\left(p^{+}-2\right) \alpha}{2}}}{\frac{\alpha\left(c_{2}\right)^{\frac{\alpha p^{+}}{2}}\left(p^{+}\right)^{\alpha}}{\frac{\bar{B}_{2} T}{r^{-}}+\left|\underline{A_{2}}\right| \sqrt{T}}} .
$$

Consider $u \in X$ such that $u(k)>1, \quad$ for $k \in \mathbb{N}[1, T]$; by (2.5), (2.6) and (2.7)

$$
J_{\lambda}(u) \leq \frac{D_{2} C_{1}^{\alpha}}{\alpha}\left[\sum_{k=1}^{T+1}\left(|\Delta u(k-1)|+\frac{|\Delta u(k-1)|^{p(k-1)}}{p^{-}}\right)\right]^{\alpha}-\lambda\left(-\left|\underline{A_{1}}\right| \sum_{k=1}^{T}|u(k)|+\frac{B_{1}}{r^{+}} \sum_{k=1}^{T}|u(k)|^{r(k)}\right) .
$$

Let $u_{t} \in X \quad$ defined in the following way $: u_{t}(k)=t$ for $k \in \mathbb{N}[1, T+1]$.

Then $t>1$, we have

$$
\begin{aligned}
J_{\lambda}\left(u_{t}\right) & \leq \frac{D_{2} C_{1}^{\alpha}}{\alpha}\left(t+\frac{t^{p(0)}}{p^{-}}\right)^{\alpha}-\lambda T\left(-\left|\underline{A_{1}}\right| t+\frac{B_{1}}{r^{+}} t^{r^{-}}\right) \\
& \leq \frac{\left(2 C_{1}\right)^{\alpha} D_{2} t^{\alpha p^{+}}}{\alpha}-\lambda T\left(-\left|\underline{A_{1}}\right| t+\frac{B_{1}}{r^{+}} t^{r^{-}}\right) .
\end{aligned}
$$

Since $r^{-}>\alpha p^{+}, \lim _{t \rightarrow+\infty} J_{\lambda}\left(u_{t}\right)=-\infty$; then there exists $t_{0}$ such that for

$$
u_{t_{0}} \in X \backslash \Omega, \quad J_{\lambda}\left(u_{t_{0}}\right)<\min _{u \in \partial \Omega} J_{\lambda}(u) .
$$

$J_{\lambda} \in C^{1}(X, \mathbb{R})$, and according to Lemma 2.2, the problem (1.1) has at least one weak nontrivial solution. 


\section{$3.3 \quad$ Case $\alpha p^{-}>r^{-}$}

In this sub section, by applying the Ekeland's variational principle we will show that the problem (1.1) admits at least a weak nontrivial solution.

Proof. Take $\lambda \in\left(0, \lambda_{1}\right)$. By proof of Theorem 3.2, for every $u \in \partial \Omega$ we have $J_{\lambda}(u)>0$.

Using Weierstrass theorem we obtain

$$
\inf _{u \in \partial \Omega} J_{\lambda}(u)>0
$$

Taking $u(k) \in(0, \beta)$, we have

$J_{\lambda}(u) \leq \frac{D_{2} C_{1}^{\alpha}}{\alpha}\left[\sum_{k=1}^{T+1}\left(|\Delta u(k-1)|+\frac{|\Delta u(k-1)|^{p(k-1)}}{p^{-}}\right)\right]^{\alpha}-\lambda\left(-\left|\underline{A_{1}}\right| \sum_{k=1}^{T}|u(k)|+\frac{B_{1}}{r^{+}} \sum_{k=1}^{T}|u(k)|^{r(k)}\right)$.

For $t \in(0, \beta)$, assume that

$$
t<\alpha^{p^{-}-r^{-}} \sqrt{\frac{\lambda\left(-\max \left(\alpha_{1}, \alpha_{2}\right)\left|\underline{A_{1}}\right|+\frac{B_{1}}{r^{+}}\right)}{\frac{D_{2}\left(2 C_{1}\right)^{\alpha}}{\alpha}\left(\beta_{1}+\frac{1}{p^{-}}\right)}},
$$

we choose $k_{0} \in \mathbb{N}[1, T]$ such that $r\left(k_{0}\right)=r^{-}$. Let $u_{0} \in X$ be a function such that $u_{0}\left(k_{0}\right)=t$, $u_{0}(k)=0$ for any $k \in \mathbb{N}[1, T] \backslash\left\{k_{0}\right\}$.

We obtain

$$
\begin{aligned}
J_{\lambda}\left(u_{0}\right) & \leq \frac{D_{2} C_{1}^{\alpha}}{\alpha}\left(2 t+\frac{t^{p\left(k_{0}-1\right)}+t^{p\left(k_{0}\right)}}{p^{-}}\right)^{\alpha}-\lambda\left(-\underline{A_{1}} \mid t+\frac{B_{1}}{r^{+}} t^{r^{-}}\right) \\
& \leq \frac{D_{2}\left(2 C_{1}\right)^{\alpha}}{\alpha}\left(t+\frac{t^{p^{-}}}{p^{-}}\right)^{\alpha}-\lambda\left(-\underline{A_{1}} \mid t+\frac{\underline{B_{1}}}{r^{+}} t^{r^{-}}\right) .
\end{aligned}
$$

There exists $\beta_{1}, \alpha_{1}>1$ such that $\beta_{1} t^{p^{-}} \geq t$ and $\alpha_{1} t^{r^{-}} \geq t$.

We have

$$
\begin{aligned}
J_{\lambda}\left(u_{0}\right) & \leq \frac{D_{2}\left(2 C_{1}\right)^{\alpha} t^{\alpha p^{-}}}{\alpha}\left(\beta_{1}+\frac{1}{p^{-}}\right)-\lambda\left(-\alpha_{1}\left|\underline{A_{1}}\right|+\frac{B_{1}}{r^{+}}\right) t^{r^{-}} \\
& \leq \frac{D_{2}\left(2 C_{1}\right)^{\alpha} t^{\alpha p^{-}}}{\alpha}\left(\beta_{1}+\frac{1}{p^{-}}\right)-\lambda\left(-\max \left(\alpha_{1}, \alpha_{2}\right)\left|\underline{A_{1}}\right|+\frac{B_{1}}{r^{+}}\right) t^{r^{-}}<0 .
\end{aligned}
$$

Thus $J_{\lambda}\left(u_{0}\right)<0$ for $u_{0} \in \operatorname{Int} \Omega$.

Therefore,

$$
\inf _{u \in I n t \Omega} J_{\lambda}(u)<0
$$

So,

$$
\inf _{u \in \operatorname{Int} \Omega} J_{\lambda}(u)<\inf _{u \in \partial \Omega} J_{\lambda}(u)
$$

Using the proof of [7] we have

$$
0<\varepsilon<\inf _{u \in \partial \Omega} J_{\lambda}(u)-\inf _{u \in \operatorname{Int} \Omega} J_{\lambda}(u) .
$$

Applying Ekeland's variationnal principle to the functional $J_{\lambda}: \Omega \longrightarrow \mathbb{R}$ we find $u_{\varepsilon} \in \Omega$ such that

$$
\begin{aligned}
J_{\lambda}\left(u_{\varepsilon}\right) & <\inf _{u \in \Omega} J_{\lambda}(u)+\varepsilon \\
& <J_{\lambda}(u)+\varepsilon\left\|u-u_{\varepsilon}\right\| \quad \text { for } \quad u \neq u_{\varepsilon} .
\end{aligned}
$$

Since

$$
J_{\lambda}\left(u_{\varepsilon}\right)<\inf _{u \in \Omega} J_{\lambda}(u)+\varepsilon \leq \inf _{u \in \operatorname{Int} \Omega} J_{\lambda}(u)+\varepsilon<\inf _{u \in \partial \Omega} J_{\lambda}(u),
$$


we deduce $u_{\varepsilon} \in$ Int $\Omega$.

Now we define $L_{\lambda}: \Omega \longrightarrow \mathbb{R}$ by

$$
L_{\lambda}(u)=J_{\lambda}(u)+\varepsilon\left\|u-u_{\varepsilon}\right\| \text { for } \quad u \neq u_{\varepsilon} .
$$

We have $u_{\varepsilon}$ as a minimum of $L_{\lambda}$ and therefore

$$
\frac{L_{\lambda}\left(u_{\varepsilon}+t v\right)-L_{\lambda}\left(u_{\varepsilon}\right)}{t}+\varepsilon\|v\| \geq 0
$$

for any $v \in \Omega$ and a small enough positive $t$.

We deduce that

$$
\frac{J_{\lambda}\left(u_{\varepsilon}+t v\right)-J_{\lambda}\left(u_{\varepsilon}\right)}{t}+\varepsilon\|v\| \geq 0 .
$$

Letting $t \rightarrow 0$, it follows that

$$
\left\langle J_{\lambda}^{\prime}\left(u_{\varepsilon}\right), v\right\rangle+\varepsilon\|v\|>0,
$$

we obtain

$$
\left\|J_{\lambda}^{\prime}\left(u_{\varepsilon}\right)\right\| \leq \varepsilon .
$$

There exists a sequence $\left\{s_{n}\right\} \subset$ Int $\Omega$ such that

$$
J_{\lambda}\left(s_{n}\right) \rightarrow \inf _{u \in \Omega} J_{\lambda}(u) \quad \text { and } \quad J_{\lambda}^{\prime}\left(s_{n}\right) \rightarrow 0
$$

Since $\left\{s_{n}\right\}$ is bounded in $X$ there exists $s_{0} \in X$ such that, up to a subsequence, $\left\{s_{n}\right\}$ converges to $s_{0} \in X$. Thus

$$
J_{\lambda}\left(s_{0}\right)=\inf _{u \in \Omega} J_{\lambda}(u) \quad \text { and } \quad J_{\lambda}^{\prime}\left(s_{0}\right)=0 .
$$

$s_{0}$ is one weak nontrivial solution for problem (1.1).

\subsection{Multiple Solutions}

In this section we prove the existence of at least two weak nontrivial solutions of the problem (1.1).

Theorem 3.3. Assume that (2.4), (2.6),(2.7), (2.10), (2.11), (2.13) holds; let $r^{-}>\alpha p^{+}$and $\tau>1$. For any $\lambda \in\left(0, \frac{\sigma_{1} \tau^{2}+\frac{D_{1}}{\left(p^{+}\right)^{\alpha-1}}\left(\frac{C_{2} \tau^{p^{-}}}{\left(c_{2}\right)^{\frac{p^{-}}{2}}}-C_{3}\right)^{\alpha}}{\tau^{r^{+}}\left(\underline{\left.\left|A_{2}\right| \sqrt{T}+2 T \overline{B_{2}}\right)}\right.}\right)$ the problem (1.1) has at least two weak nontrivial solutions where one solution satisfies $\|u\|>1$.

Proof. Let

$$
\Omega_{\tau}:=\{u \in X:\|u\| \leq \tau\} ; \quad \Omega_{\chi}:=\{u \in X:\|u\| \geq \chi\},
$$

where $\chi \in(1, \tau)$. Assume that $u_{0} \in X$ is a local minimizer of $J_{\lambda}$ in $\Omega=\Omega_{\tau} \cap \Omega_{\chi}$.

If $u_{0} \in \operatorname{Int}(\Omega)$ by using Lemma $2.2 \quad J_{\lambda}\left(u_{0}\right)<\min _{u \in \partial \Omega_{\tau}} J_{\lambda}(u)$.

Now suppose that $u_{0} \in \partial \Omega_{\tau}$, by Theorem 2.3 there exist $\sigma_{0}, \sigma_{1}, \sigma_{2} \geq 0 ; \sigma_{0}^{2}+\sigma_{1}^{2}+\sigma_{2}^{2}>0$ such that for all $v \in X$

$$
\sigma_{1}\left(\left\|u_{0}\right\|^{2}-\tau^{2}\right)=0, \quad \sigma_{2}\left(\chi^{2}-\left\|u_{0}\right\|^{2}\right)=0
$$

and

$$
\sigma_{0}\left\langle J_{\lambda}^{\prime}\left(u_{0}\right), v\right\rangle+\sigma_{1}\left\langle u_{0}, v\right\rangle-\sigma_{2}\left\langle u_{0}, v\right\rangle=0 .
$$

Since $u_{0} \in \partial \Omega_{\tau}$, we have $\left\|u_{0}\right\|=\tau$ and $\sigma_{2}=0$. Taking $v=u_{0}$ and $\sigma_{0}=1$ we see that $M\left(\sum_{k=1}^{T+1} A\left(k-1, \Delta u_{0}(k-1)\right)\right) \sum_{k=1}^{T+1} a\left(k-1, \Delta u_{0}(k-1)\right) \Delta u_{0}(k-1)+\sigma_{1}\left\|u_{0}\right\|^{2}=\lambda \sum_{k=1}^{T} f\left(k, u_{0}(k)\right) u_{0}(k)$. 
By (2.4), (2.6), (2.11) and (2.13)

$$
\begin{array}{r}
M\left(\sum_{k=1}^{T+1} A\left(k-1, \Delta u_{0}(k-1)\right)\right) \sum_{k=1}^{T+1} a\left(k-1, \Delta u_{0}(k-1)\right) \Delta u_{0}(k-1)+\sigma_{1}\left\|u_{0}\right\|^{2} \geq \sigma_{1} \tau^{2}+ \\
D_{1}\left(\sum_{k=1}^{T+1} A\left(k-1, \Delta u_{0}(k-1)\right)\right) \sum_{k=1}^{\alpha-1} a\left(k-1, \Delta u_{0}(k-1)\right) \Delta u_{0}(k-1) .
\end{array}
$$

We have

$$
\begin{gathered}
M\left(\sum_{k=1}^{T+1} A\left(k-1, \Delta u_{0}(k-1)\right)\right) \sum_{k=1}^{T+1} a\left(k-1, \Delta u_{0}(k-1)\right) \Delta u_{0}(k-1)+\sigma_{1}\left\|u_{0}\right\|^{2} \geq \sigma_{1} \tau^{2}+ \\
\frac{D_{1}}{\left(p^{+}\right)^{\alpha-1}}\left(\frac{C_{2} \tau^{p^{-}}}{\left(c_{2}\right)^{\frac{p^{-}}{2}}}-C_{3}\right)^{\alpha-1} \sum_{k=1}^{T+1}\left|\Delta u_{0}(k-1)\right|^{p(k-1)} .
\end{gathered}
$$

We obtain

$$
\begin{gathered}
M\left(\sum_{k=1}^{T+1} A\left(k-1, \Delta u_{0}(k-1)\right)\right) \sum_{k=1}^{T+1} a\left(k-1, \Delta u_{0}(k-1)\right) \Delta u_{0}(k-1)+\sigma_{1}\left\|u_{0}\right\|^{2} \geq \sigma_{1} \tau^{2}+ \\
\frac{D_{1}}{\left(p^{+}\right)^{\alpha-1}}\left(\frac{C_{2} \tau^{p^{-}}}{\left(c_{2}\right)^{\frac{p^{-}}{2}}}-C_{3}\right)^{\alpha} .
\end{gathered}
$$

Using (2.7) and (2.10)

$$
\begin{aligned}
\lambda \sum_{k=1}^{T} f\left(k, u_{0}(k)\right) u_{0}(k) & \leq \lambda\left(\left|\underline{\mid A_{2}}\right| \sum_{k=1}^{T}\left|u_{0}(k)\right|+\overline{B_{2}} \sum_{k=1}^{T}\left|u_{0}(k)\right|^{r^{+}}+\overline{B_{2}} \sum_{k=1}^{T}\left|u_{0}(k)\right|^{r^{-}}\right) \\
& \leq \lambda\left(\left.\left|\underline{A_{2} \mid} \sqrt{T}\right|\left|u_{0}\right|\left|+T \overline{B_{2}}\right|\left|u_{0}\right|\right|^{r^{+}}+T \overline{B_{2}}|| u_{0}||^{r^{-}}\right) \\
& \leq \lambda \tau^{r^{+}}\left(\left|\underline{A_{2}}\right| \sqrt{T}+2 T \overline{B_{2}}\right) .
\end{aligned}
$$

So,

$$
\sigma_{1} \tau^{2}+\frac{D_{1}}{\left(p^{+}\right)^{\alpha-1}}\left(\frac{C_{2} \tau^{p^{-}}}{\left(c_{2}\right)^{\frac{p^{-}}{2}}}-C_{3}\right)^{\alpha} \leq \lambda \tau^{r^{+}}\left(\underline{\mid A_{2}} \mid \sqrt{T}+2 T \overline{B_{2}}\right)
$$

This is contradictory. Hence $u_{0} \in \operatorname{Int}(\Omega)$ is a nontrivial minimizer of $J_{\lambda}$.

By proof of Theorem (3.2) there exists $u_{1} \in X \backslash \Omega$ such that $J_{\lambda}\left(u_{1}\right)<\min _{u \in \partial \Omega_{\tau}} J_{\lambda}(u)$.

By Lemma $2.2 \quad u^{*} \in X$ is critical point of the functional $J_{\lambda}$.

We have $u_{0}$ and $u^{*}$ are two different weak nontrivial solution of the prolem (1.1) and since $u_{0} \in \operatorname{Int}(\Omega)$, it is easy to see that $\left\|u_{0}\right\|>1$.. 


\section{An Extension}

In this section, we show that the existence result obtained in (1.1) can be extended to more general discrete boundary value problems of the form

$$
\left\{\begin{array}{l}
-M(A(k-1, \Delta u(k-1))) \Delta(a(k-1, \Delta u(k-1)))+|u|^{q(k)-2} u(k)=\lambda f(k, u(k)), k \in \mathbb{N}[1, T] \\
u(0)=\Delta u(T)=0,
\end{array}\right.
$$

with $q: \mathbb{N}[1, T] \longrightarrow(2,+\infty)$.

A function $u \in X$ is a solution of problem (4.1) if for any $v \in X$,

$M\left(\sum_{k=1}^{T+1} A(k-1, \Delta u(k-1))\right) \sum_{k=1}^{T+1} a(k-1, \Delta u(k-1)) \Delta v(k-1)+\sum_{k=1}^{T}|u(k)|^{q(k)-2} u(k) v(k)=\lambda \sum_{k=1}^{T} f(k, u(k)) v(k)$.

Theorem 4.1. Assume that $\alpha p^{-}>r^{+}$and $\lambda>\lambda_{2}$, the problem (4.1) has at least one weak nontrivial solution .

\section{Proof.}

In this part we use the proof of Theorem 3.1. For $u \in X$, we define the energy functional $J$ by

$$
J_{\lambda}(u)=\widehat{M}\left(\sum_{k=1}^{T+1} A(k-1, \Delta u(k-1))\right)+\sum_{k=1}^{T} \frac{1}{q(k)}|u|^{q(k)}-\lambda \sum_{k=1}^{T} F(k, u(k)) .
$$

The functional $J$ is well defined, weakly lower semi continuous and is of class $C^{1}(X, \mathbb{R})$ with a derivative given by

$$
\begin{aligned}
\left\langle J_{\lambda}^{\prime}(u), v\right\rangle= & M\left(\sum_{k=1}^{T+1} A(k-1, \Delta u(k-1))\right) \sum_{k=1}^{T+1} a(k-1, \Delta u(k-1)) \Delta v(k-1)+ \\
& \sum_{k=1}^{T}|u(k)|^{q(k)-2} u(k) v(k)-\lambda \sum_{k=1}^{T} f(k, u(k)) v(k),
\end{aligned}
$$

for all $u, v \in X$.

Since

$$
\sum_{k=1}^{T} \frac{1}{q(k)}|u|^{q(k)} \geq 0
$$

we have

$$
J_{\lambda}(u) \geq \widehat{M}\left(\sum_{k=1}^{T+1} A(k-1, \Delta u(k-1))\right)-\lambda \sum_{k=1}^{T} F(k, u(k)) .
$$

According to Proposition (3.1) we deduce that $J_{\lambda}$ is coercive. Let $u_{\lambda}$ be a global minimizer of $J_{\lambda}$, taking $u_{0}$ such that $u_{0}\left(k_{0}\right)=t_{0}, \quad u_{0}(k)=0$, for $k \in \mathbb{N}[0, T] \backslash\left\{k_{0}\right\}$ and $u_{0}(0)=\Delta u_{0}(T)=0$ with $t_{0}>1$ is a fixed real.

We have

$$
J_{\lambda}\left(u_{0}\right) \leq \frac{D_{2}\left(4 C_{1}\right)^{\alpha} t_{0}^{\alpha p^{+}}}{\alpha}+\frac{t_{0}^{q^{+}}}{q^{-}}-\lambda t_{0}\left(-\max \left(\alpha_{1}, \alpha_{2}\right)\left|\underline{A_{1}}\right|+\frac{\underline{B_{1}}}{r^{+}}\right)
$$

where

$$
\lambda_{2}=\frac{\frac{D_{2}\left(4 C_{1}\right)^{\alpha} t_{0}^{\alpha p^{+}}}{\alpha}+\frac{t_{0}^{q^{+}-1}}{q^{-}}}{-\max \left(\alpha_{1}, \alpha_{2}\right)\left|\underline{A_{1}}\right|+\frac{B_{1}}{r^{+}}} .
$$

It follows that $J_{\lambda}\left(u_{\lambda}\right)<0$ for any $\lambda>\lambda_{2} . u_{\lambda}$ is one weak nontrivial solution of problem (4.1). 
Lemma 4.1. Assume that (2.10) hold and $r^{-}>\max \left(\alpha p^{+}, q^{+}\right)$. Then for any $\lambda>0$ the functional $J_{\lambda}$ satisfies the Palais-Smale condition.

Proof. By the proof of Lemma 3.1, we obtain

$$
\begin{aligned}
J_{\lambda}\left(u_{n}\right) \leq \frac{D_{2} C_{1}^{\alpha}}{\alpha}\left[\left|u_{n}(T+1)\right|+2 \sqrt{T}|| u_{n}||+\frac{(T+1)\left(2^{p^{+}} \| u_{n}||^{p^{+}}+1\right)}{p^{-}}\right]^{\alpha}+ \\
\sum_{k=1}^{T} \frac{1}{q(k)}\left|u_{n}\right|^{q(k)}-\lambda\left(-\left|\underline{A_{1}}\right| \sqrt{T}|| u_{n}\left\|^{2}+\frac{B_{1} T^{\frac{2-r^{-}}{2}}}{r^{+}}\right\| u_{n}||^{r^{-}}\right) .
\end{aligned}
$$

By $(2.10)$

$$
\begin{gathered}
J_{\lambda}\left(u_{n}\right) \leq \frac{D_{2} C_{1}^{\alpha}}{\alpha}\left[\left|u_{n}(T+1)\right|+2 \sqrt{T}|| u_{n} \|+\frac{(T+1)\left(2^{p^{+}}\left\|u_{n}\right\|^{p^{+}}+1\right)}{p^{-}}\right]^{\alpha}+ \\
\frac{\left.T\left\|u_{n}\right\|\right|^{q^{+}}}{q^{-}}-\lambda\left(-\mid \underline{\left.A_{1}|\sqrt{T}| \mid u_{n}\left\|^{2}+\frac{B_{1} T^{\frac{2-r^{-}}{2}}}{r^{+}}\right\| u_{n} \|^{r^{-}}\right) .}\right.
\end{gathered}
$$

Since $r^{-}>\max \left(\alpha p^{+}, q^{+}\right)$then the sequence $\left\{u_{n}\right\}$ is bounded.

In this part we apply the mountain pass geometry Lemma.

Theorem 4.2. Assume that $r^{-}>\max \left(\alpha p^{+}, q^{+}\right)$holds. Then for $\lambda \in\left(0, \lambda_{1}\right)$ the problem (4.1) has at least one weak nontrivial solution.

Proof. We will refer to the proof of theorem 3.2 .

Let

$$
\Omega=\{u \in X:\|u\| \leq \theta\}
$$

with $\theta \in(0,1)$.

For $u \in \Omega$

$$
J_{\lambda}(u) \geq \frac{D_{1} T^{-\frac{\left(p^{+}-2\right) \alpha}{2}}}{\alpha\left(c_{2}\right)^{\frac{\alpha p^{+}}{2}}\left(p^{+}\right)^{\alpha}} \theta^{\alpha p^{+}}-\lambda \theta\left(\frac{\overline{B_{2} T}}{r^{-}}+\left|\underline{A_{2}}\right| \sqrt{T}\right) .
$$

For all $\lambda \in\left(0, \lambda_{1}\right)$ we obtain $\inf _{u \in \partial \Omega} J_{\lambda}(u)>0$. Let $u_{t} \in X$ be defined as follows : $u_{t}(k)=t$ for $k \in \mathbb{N}[1, T+1]$.

For $t>1$, we have

$$
J_{\lambda}(u) \leq \frac{\left(2 C_{1}\right)^{\alpha} D_{2} t^{\alpha p^{+}}}{\alpha}+T t^{q^{+}}-\lambda T\left(-\left|\underline{A_{1}}\right| t+\frac{B_{1}}{r^{+}} t^{r^{-}}\right)
$$

Since $r^{-}>\max \left(\alpha p^{+}, q^{+}\right), \lim _{t \rightarrow+\infty} J_{\lambda}\left(u_{t}\right)=-\infty$; then it exist $t_{1}$ such that

$$
u_{t_{1}} \in X \backslash \Omega, \quad J_{\lambda}\left(u_{t_{1}}\right)<\min _{u \in \partial \Omega} J_{\lambda}(u) .
$$

$J_{\lambda} \in C^{1}(X, \mathbb{R})$, and the assumption of Lemma 2.2 the problem (4.1) has at least one weak nontrivial solution.

We apply Ekeland's variational principe with $\min \left(\alpha p^{-}, q^{-}\right)>r^{-}$, we will use the result of case $\alpha p^{-}>r^{-}$. 
For $\lambda \in\left(0, \lambda_{1}\right)$

$$
\inf _{u \in \partial \Omega} J_{\lambda}(u)>0
$$

For $t \in(0, \theta)$, assume that

$$
t<\min \left(\alpha p^{-}, q^{-}\right)-r^{-} \sqrt{\frac{\lambda\left(-\max \left(\alpha_{1}, \alpha_{2}\right)\left|\underline{A_{1}}\right|+\frac{B_{1}}{r^{+}}\right)}{\frac{D_{2}\left(2 C_{1}\right)^{\alpha}}{\alpha}\left[\left(\beta_{2}+\frac{1}{p^{-}}\right)^{\alpha}+\frac{\alpha}{D_{2}\left(2 C_{1}\right)^{\alpha}}\right]}},
$$

we choose $k_{0} \in \mathbb{N}[1, T]$ such that $r\left(k_{0}\right)=r^{-}$. Let $u_{0} \in X$ be a function such that $u_{0}\left(k_{0}\right)=t$ and $u_{0}(k)=0$, for any $k \in \mathbb{N}[1, T] \backslash\left\{k_{0}\right\}$.

We obtain

$$
J_{\lambda}\left(u_{0}\right) \leq \frac{D_{2}\left(2 C_{1}\right)^{\alpha}}{\alpha}\left(t+\frac{t^{p^{-}}}{p^{-}}\right)^{\alpha}+t^{q^{-}}-\lambda\left(-\underline{A_{1}} \mid t+\frac{\underline{B_{1}}}{r^{+}} t^{r^{-}}\right)
$$

There exists $\beta_{2}, \alpha_{2}>1$ such that $\beta_{2} t^{p^{-}} \geq t$ and $\alpha_{2} t^{r^{-}} \geq t$.

We have

$$
\begin{aligned}
J_{\lambda}\left(u_{0}\right) & \leq \frac{D_{2}\left(2 C_{1}\right)^{\alpha} t^{\alpha p^{-}}}{\alpha}\left(\beta_{2}+\frac{1}{p^{-}}\right)^{\alpha}+t^{q^{-}}-\lambda\left(-\left|\underline{A_{1}}\right| t+\frac{B_{1}}{\overline{r^{+}}} t^{r^{-}}\right) \\
& \leq \frac{D_{2}\left(2 C_{1}\right)^{\alpha} t^{\min \left(\alpha p^{-}, q^{-}\right)}}{\alpha}\left[\left(\beta_{2}+\frac{1}{p^{-}}\right)^{\alpha}+\frac{\alpha}{D_{2}\left(2 C_{1}\right)^{\alpha}}\right]-\lambda\left(-\alpha_{2}\left|\underline{A_{1}}\right|+\frac{B_{1}}{\overline{r^{+}}}\right) t^{r^{-}} \\
& \leq \frac{D_{2}\left(2 C_{1}\right)^{\alpha} t^{\min \left(\alpha p^{-}, q^{-}\right)}}{\alpha}\left[\left(\beta_{2}+\frac{1}{p^{-}}\right)^{\alpha}+\frac{\alpha}{D_{2}\left(2 C_{1}\right)^{\alpha}}\right]-\lambda\left(-\max \left(\alpha_{1}, \alpha_{2}\right)\left|\underline{A_{1}}\right|+\frac{B_{1}}{\overline{r^{+}}}\right) t^{r^{-}}<0
\end{aligned}
$$

Thus, $J_{\lambda}\left(u_{0}\right)<0$ for $u_{0} \in \operatorname{Int} \Omega$.

By the same reasoning we prove that the problem (4.1) has at least one weak nontrivial solution.

Now we will prove that problem (4.1) has at least two weak nontrivial solution.

In the case of multiple solutions we will use the Theorem 3.3 and the same sets defined previously.

Proof. Let $u_{0} \in X$ a local minimizer of $J_{\lambda}$ on $\Omega=\Omega_{\tau} \cap \Omega_{\chi}$. If $u_{0} \in \operatorname{Int}(\Omega)$ we have $J_{\lambda}\left(u_{0}\right)<\min _{u \in \partial \Omega_{\tau}} J_{\lambda}(u)$.

Assume that $u_{0} \in \partial \Omega_{\tau}$, we have $\sigma_{2}=0$, taking $v=u_{0}$ and $\sigma_{0}=1$ we get

$$
\begin{array}{r}
M\left(\sum_{k=1}^{T+1} A\left(k-1, \Delta u_{0}(k-1)\right)\right) \sum_{k=1}^{T+1} a\left(k-1, \Delta u_{0}(k-1)\right) \Delta u_{0}(k-1)+\sum_{k=1}^{T}\left|u_{0}\right|^{q(k)}+ \\
\sigma_{1}\left\|u_{0}\right\|^{2}=\lambda \sum_{k=1}^{T} f\left(k, u_{0}(k)\right) u_{0}(k) .
\end{array}
$$

We have

$$
\begin{gathered}
M\left(\sum_{k=1}^{T+1} A\left(k-1, \Delta u_{0}(k-1)\right)\right) \sum_{k=1}^{T+1} a\left(k-1, \Delta u_{0}(k-1)\right) \Delta u_{0}(k-1)+\sum_{k=1}^{T}\left|u_{0}\right|^{q(k)}+\sigma_{1}|| u_{0} \|^{2} \geq \\
M\left(\sum_{k=1}^{T+1} A\left(k-1, \Delta u_{0}(k-1)\right)\right) \sum_{k=1}^{T+1} a\left(k-1, \Delta u_{0}(k-1)\right) \Delta u_{0}(k-1)+\sigma_{1}\left\|u_{0}\right\|^{2} .
\end{gathered}
$$

By the same reasoning we prove that problem (4.1) has at least two weak nontrivial solutions.

Acknowledgments. The authors express their deepest thanks to the editor and anonymous referee for their comments and suggestions on the article. 


\section{References}

1. S.N. Elaydi, An Introduction to Difference Equations, Undergraduate Texts in Mathematics, SpringerVerlag,New York, 1999.

2. V. Lakshmikantham and D. Trigiante, Theory of Difference Equations: Numerical Methods and Applications, Academic Press, New York, 1988.

3. D.W. Oplinger; Frequency response of a nonlinear stretched string, J. Acoustic Soc. Amer 32(1960), 15291538.

4. Blaise Koné and Stanislas Ouaro; On the Solvability of Discrete Nonlinear Two-Point Boundary Value Problems International Journal of Mathematics and Mathematical Sciences; Vol. 2012.

5. Blaise Koné, Ismael Nyanquini and Stanislas Ouaro; Weak Solutions to Discrete Nonlinear Two-Point Boundary-Value Problems of Kirchhoff Type, Electronic Journal of Differential Equations, Vol. 2015(2015), No. 105, pp. 1-10.

6. X. Cai and J. Yu; Existence theorems for second-order discrete boundary value problems, J. Math. Anal. Appl. 320 (2006), 649-661.

7. M. Mihailescu, V. Radulescu and S. Tersian; Eigenvalue problems for anisotropic discrete boundary value problems, J. Differ. Equ. Appl. 15 (2009), 557-567.

8. Marek Galewski and Renata Wieteska.Multiple periodic solutions to a discrete $p(k)$ - Laplacian problem.

9. J. Mawhin, Problèmes de Dirichlet variationnels non linéaires, Les Presses de l'Université de Montréal, 1987.

10. De Figueiredo DG. Lectures on the Ekeland Variational Principe with Applications and Detours. Bombay: Tata Institute of Fundamental Research, 1989.

11. Dajun G. Nonlinear Functional Analysis. Shandong Science and Technology Press, 1985.

12. Borwein JM, Lewis As. Convex analysis and nonlinear optimization. Theory and examples. 2nd ed., CMS Books in Mathematics/Ouvrages de Mathématiques de la SMC 3. New York, NY: Springer. Xii, 2006.

13. Rodrigue Sanou, Idrissa Ibrango,Blaise Koné and Aboudramane Guiro; Weak Solutions to Neumann discrete nonlinear System of Kirchhoff type, Cubo A Mathematical Journal, Vol.21, num 03, (75-91). December 2019.

14. Idrissa Ibrango, Rodrigue Sanou, Blaise Koné and Aboudramane Guiro; Weak Homoclinic Solutions of Anisotropic Discrete nonlinear System with Variable Exponent, Nonauton.Dyn.Syst.2020. 